\title{
Evolution of the Size Distribution of Oil-droplets Over Time in Oil-in-water Emulsions
}

\author{
Kazutaka KATSUKI $^{1}$, Yayoi MIYAGAWA ${ }^{1}$, Ryuichi MATSUNO ${ }^{2}$, Shuji ADACHI ${ }^{1, \dagger}$ \\ ${ }^{1}$ Division of Food Science and Biotechnology, Graduate School of Agriculture, Kyoto University, \\ Sakyo-ku, Kyoto 606-8502, Japan \\ ${ }^{2}$ Professor Emeritus of Ishikawa Prefectural University and Kyoto University
}

\begin{abstract}
A theoretical method to calculate the evolution of the size distribution of oil-droplets in $\mathrm{O} / \mathrm{W}$ emulsions over time was proposed based on the model where the coalescence rate of two oil-droplets was expressed by second-order kinetics using the rate constant evaluated from the activation energy for coalescence. The force between two oil-droplets of different diameters was appropriated by the force between two oil-droplets of equivalent diameter, and the increase in the rate of the number density of oil-droplets was estimated by solving the mass balance equation. The evolution of the size distribution of oil-droplets in several conditions agreed well with well-known features of coalescence of oil-droplets in O/W emulsion such as instability with larger oil-droplet size.
\end{abstract}

Keywords: emulsion, coalescence, oil-droplet size, size distribution, kinetics

\section{Introduction}

Oil-in-water $(\mathrm{O} / \mathrm{W})$ emulsions, which are widely used in the food, cosmetic, and pharmaceutical industries, are thermodynamically unstable or pseudo-stable. However, the preparation of highly stable emulsions is important for industrial applications, and many experimental efforts have been expended for improving the stability of these emulsions [1]. It is empirically known that emulsions containing small oil-droplets are more stable. Since the stability of $\mathrm{O} / \mathrm{W}$ emulsions is related to the size of the oil-droplets, theoretical as well as experimental studies have been conducted to predict the size distribution of the oil-droplets in the $\mathrm{O} / \mathrm{W}$ emulsions prepared under various emulsification conditions [2-4]. Theoretical predictions of the size distribution facilitate reasonable design of the emulsification process, and enable us to predict the effects of some factors, such as the viscosity of the continuous phase, mean oil-droplet size, and size distribution, on the stability. Conversely, prediction of the destabilization process is expected to provide the knowledge required to prepare stable emulsions. A few studies on predicting or calculating the changes in the size distribution of the oil-droplets in the cream layer of dense $\mathrm{O} /$ $\mathrm{W}$ emulsions have been reported [5].

In many cases, the coalescence rate of the oil-droplets

(Received 25 May. 2015: accepted 14 Jul. 2015)

$\dagger$ Fax: +81-75-753-6285, E-mail: adachi@kais.kyoto-u.ac.jp is treated by second-order kinetics [6]. In order to analytically calculate the change in the size distribution of the oil-droplets based on the kinetics, we need to determine the rate constant for the coalescence of oil-droplets of various sizes. When the activation energy for coalescence is known, we can estimate the rate constant. Previously, we [7] proposed a model to estimate the effects of various parameters, such as oil-droplet size, surface potential of the oil-droplets, and viscosity of the continuous phase, on the stability of dense $\mathrm{O} / \mathrm{W}$ emulsions based on the DLVO theory [8] and Stokes' law. The model was able to successfully predict that emulsions with smaller oil-droplets were more stable than those with larger oil-droplets, which was consistent with our experience. We have recently modified the model to enable the prediction of activation energy for the coalescence of oil-droplets of any size [9].

In this study, we propose a theoretical method to calculate the evolution of the size distribution of oil-droplets in $\mathrm{O} / \mathrm{W}$ emulsions over time, based on the modified model.

\section{Theoretical Considerations}

The coalescence rate of two oil-droplets of diameters $D_{i}$ and $D_{j}, R\left(D_{i}, D_{j}\right)$, can be expressed by second-order kinetics as follows.

$$
R\left(D_{i}, D_{j}\right)=k\left(D_{i}, D_{j}\right) C\left(D_{i}\right) C\left(D_{j}\right)
$$


where $C\left(D_{i}\right)$ and $C\left(D_{j}\right)$ are the number densities of the oil-droplets with diameters $D_{i}$ and $D_{j}$, respectively, with units of number of droplets $/ \mathrm{m}^{4}$, which is the same as the units for the frequency distribution of the oil-droplets. The rate constant $k\left(D_{i}, D_{j}\right)$ is evaluated from the activation energy for coalescence, $E\left(D_{i}, D_{j}\right)$, by Eq. (2).

$$
k\left(D_{i}, D_{j}\right)=k_{0} \exp \left[-\frac{E\left(D_{i}, D_{j}\right)}{R T}\right]
$$

The force acting between two oil-droplets of diameters $D_{i}$ and $D_{j}$, can be approximated by the force acting between two oil-droplets of equivalent diameter, $D_{\mathrm{e}}$, which is defined by Eq. (3) [10-12].

$$
\frac{2}{D_{\mathrm{e}}}=\frac{1}{D_{i}}+\frac{1}{D_{j}}
$$

The activation energy for the coalescence of two oildroplets with diameters $D_{i}$ and $D_{j}$, may also be approximated by that for the coalescence of two oil-droplets with equivalent diameter, $D_{\mathrm{e}}$.

The value of the frequency factor for coalescence, $k_{0}$, is unknown. In the present study, we assume the value of $k_{0}$ to be 1 , in order to understand the relative change in the size distribution of the oil-droplets over time. Based on the modified model and using this assumed value of $k_{0}$, the smaller oil-droplet has a larger value of $E$, and the rate constant, $k$, tends towards 0 , compared to the larger oil-droplet, which has a smaller value of $E$, and whose rate constant tends towards 1 . Thus, the rate constant ranges between 0 and 1 , depending on the equivalent diameter of the oil-droplets. The value of $E$ for the coalescence of two oil-droplets with the equivalent diameter, $D_{\mathrm{e}}$, can be obtained from our previous study [9].

The increase in the rate of the number density of the oil-droplets with diameter $D_{i}$ is given by Eq. (4), based on the mass balance equation.

$$
\begin{aligned}
\frac{d C\left(D_{i}\right)}{d t}= & -\int_{0}^{\infty} k\left(D_{i}, D_{j}\right) C\left(D_{i}\right) C\left(D_{j}\right) d D_{j} \\
& +\int_{0}^{D_{i}} k\left(D_{j}, D_{k}\right) C\left(D_{j}\right) C\left(D_{k}\right) d D_{j}
\end{aligned}
$$

The first and second terms on the right-hand side of Eq. (4) indicate the disappearance and formation rates of oildroplets with the diameter $D_{i}$, respectively. The diameter $D_{k}$ is given by Eq. (5).

$$
D_{k}=\sqrt[3]{D_{i}^{3}-D_{j}^{3}}
$$

The number densities, $C\left(D_{i}\right)$ and $C\left(D_{j}\right)$, in Eq. (4) are normalized by the initial number density of all the oildroplets, $C_{0}$, as follows:

$$
\begin{aligned}
& \frac{d C\left(D_{i}\right) / C_{0}}{d \theta}= \\
& -\int_{0}^{\infty} \exp \left[-\frac{E\left(D_{i}, D_{j}\right)}{R T}\right] \frac{C\left(D_{i}\right)}{C_{0}} \frac{C\left(D_{j}\right)}{C_{0}} d D_{j} \\
& \quad+\int_{0}^{D_{i}} \exp \left[-\frac{E\left(D_{j}, D_{k}\right)}{R T}\right] \frac{C\left(D_{j}\right)}{C_{0}} \frac{C\left(D_{k}\right)}{C_{0}} d D_{j}
\end{aligned}
$$

where $\theta$ is the dimensionless time defined by Eq. (7).

$$
\theta=k_{0} C_{0} t
$$

In order to solve Eq. (6) easily, the smallest oil-droplet size (diameter) is first assumed. The sizes of the oildroplets that are generated by the coalescence of two, three, $\ldots n$ oil-droplets with the smallest size are considered, and the mass balance of the oil-droplet for each size is then calculated. Using this treatment, we can calculate the number densities of the oil-droplets with diameters that are $2^{1 / 3}, 3^{1 / 3}, \ldots, n^{1 / 3}$ times the diameter of the smallest oil-droplet.

\section{Results and Discussion}

For parameters other than the diameter of the oildroplet, the same values were used as those in our previous studies [7,9], Briefly, the values of some of the other parameters are as follows: Hamker's constant $A=10^{-20} \mathrm{~J}$, absolute temperature $T=298 \mathrm{~K}$, dielectric constant of the continuous phase $\varepsilon=6.90 \times 10^{-10} \mathrm{~F} / \mathrm{m}$, viscosity of the continuous phase $\eta=5.0 \times 10^{-3} \mathrm{~kg} / \mathrm{m} \cdot \mathrm{s}$, Debye's factor $\kappa=$ $1.0 \times 10^{-8} \mathrm{~m}^{-1}$, density of the oil-droplet $\rho_{\mathrm{s}}=888 \mathrm{~kg} / \mathrm{m}^{3}$, and surface potential of the oil-droplet $\psi_{0}=5.0 \times 10^{-2} \mathrm{~V}$. Under these conditions, the activation energy $E$ for the coalescence of oil-droplets with an equivalent diameter of $D_{\mathrm{e}}$ was evaluated from our previous study [9].

Figure 1(a), (b), and (c) show the changes in the size distribution of the oil-droplets over time (expressed as a dimensionless quantity), when the sizes of the smallest oil-droplets were assumed to be $0.183,0.243$, and 0.487 $\mu \mathrm{m}$, respectively. In all the cases, homogeneous oildroplets with the same sizes were assumed.

For the oil-droplet with the smallest size of $0.183 \mu \mathrm{m}$ (Figure 1(a)), the rate constant for coalescence was small because of the large activation energy. Since the coalescence rate was very slow for the small oil-droplets, the change in the size distribution was also very slow. In this case, the coalescence of small oil-droplets was the rate-limiting step, and the frequencies of the number densities of larger oil-droplets were very low. The emulsion was very stable when the oil-droplet size was 


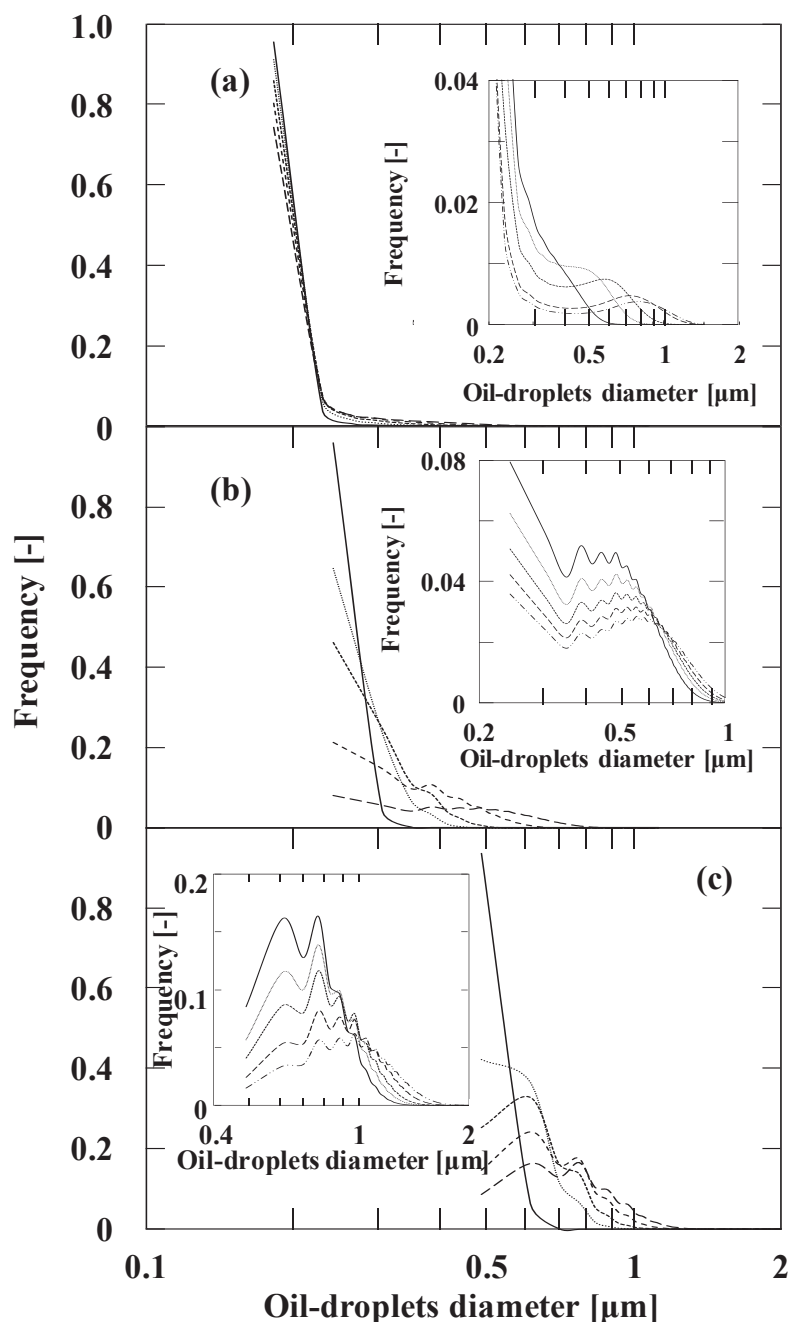

Fig. 1 Changes in the oil-droplet size distribution for $\mathrm{O} / \mathrm{W}$ emulsions having initial oil-droplet diameters of (a) $0.183 \mu$ $\mathrm{m}$, (b) $0.243 \mu \mathrm{m}$, and (c) $0.487 \mu \mathrm{m}$ at various dimensionless times, $\theta$. Insets: Magnified figures of the size distributions in the region of small oil-droplet diameters at long dimensionless times.

Dimensionless time: (a) (-) 20, (…) 40, (----) 60, (- - ) 80, (- ) 100; Inset (-) 100, (…) 160, (----) 220, ($340,(-. \cdot-) 400$, (b) (-) 0.2, (….) 2.2, (----) 4.2, (-- -) $10.2,(--) 22.2$; Inset $(-) 22.2,(\cdots \cdots) 26.2,(----) 30.2$, $(--)$ 34.2, (-..-) 38.2, (c) (-) 0.05, (…) 0.85, (-----) 1.65, (- - ) 2.85, (- -) 4.45; Inset (-) 4.45, (…) 6.05, $(----) 7.65,(--)$ 10.85, (-.--) 14.85.

smaller than $0.183 \mu \mathrm{m}$. The oil-droplet size is naturally confined under the above-mentioned conditions.

Figure 1(b) shows the change in the size distribution of the oil-droplets when the size of the smallest oil-droplet was larger than that in Fig. 1(a). The activation energy in this case was lower than that in the former case (Fig. 1(a)). The rate constant was ca. 1/10th of the maximum rate constant of 1 . Therefore, in this case, the coalescence of small oil-droplets was not rate-limiting, and the size distribution changed quickly. Although the peak in the size distribution curve remained in the region of small oil-droplet size, the peak gradually shifted towards larger oil-droplet sizes, over time. The change in the size distribution was similar to the change that was experimentally observed by Mita et al. [13].

Figure 1(c) shows the change in the size distribution of the oil-droplets in the case where the rate constant for coalescence was near the maximum value of 1 because of the very low activation energy. In this case, the change in the size distribution was much faster than those in Figs 1(a) and (b). The coalescence of small oil-droplets was not rate-limiting and the distribution in the region of small oil-droplet size lost its shape in the early stage, shifting towards larger oil-droplet sizes.

Oil-droplets with a size of $0.231 \mu \mathrm{m}$, which corresponds to the size of oil-droplets formed through the coalescence of two oil-droplets of $0.183 \mu \mathrm{m}$ (the smallest oil-droplet size), was added to the emulsion having the oil-droplet size of $0.183 \mu \mathrm{m}$ at the number density of 0.05 . The change in the size distribution of the oil-droplets was calculated to determine whether an emulsion having a homogeneous oil-droplet size distribution is more stable than that having an inhomogeneous one (Fig. 2). Although the addition of large oil-droplets showed signs of destabilizing the emulsion, its effect was

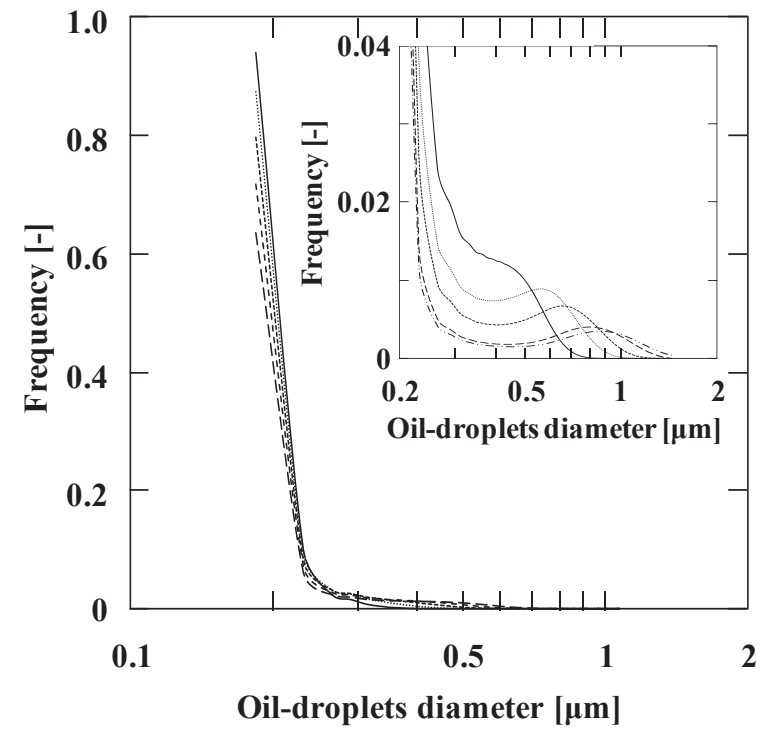

Fig. 2 Changes in the oil-droplet size distribution of $\mathrm{O} / \mathrm{W}$ emulsions where oil-droplets with a size of $0.231 \mu \mathrm{m}$ was added to the emulsion having an oil-droplet size of $0.183 \mu \mathrm{m}$ at the number density of 0.05 . Inset: Magnified figure of the distributions in the region of small oil-droplet diameters at long dimensionless times.

Dimensionless time: $(-)$ 20, (…) 40, (----) 60, (- - ) 80, $(--)$ 100; Inset $(-)$ 100, (….) 160, (----) 220, (- 340 , $(-\cdot-) 400$. 
not significant. This weak effect of the addition of large oil-droplets on the stability of the emulsion may be attributed to the small inhomogeneity in the initial size distribution of the oil-droplets.

\section{References}

1) J. L. Ortega-Vinuesa, A. Martín-Rodríguez, R. HidalgoÁlvarez; Colloidal stability of polymer colloids with different interfacial properties: Mechanisms, J. Colloid Interface Sci., 184, 259-267 (1996).

2) E. A. van der Zwan, C. G. P. H. Schroën, R. M. Boom; Premix membrane emulsification by using a packed layer of glass beads. AIChE J., 54, 2190-2197 (2008).

3) Z. Chen, J. Prüss, H. -J. Warnecke; A population balance model for disperse systems: Drop size distribution in emulsion. Chem. Eng. Sci., 53, 1059-1066 (1998).

4) N. B. Raikar, S. R. Bhatia, M. F. Malone, M. A. Henson; Experimental studies and population balance equation models for breakage prediction of emulsion drop size distribution. Chem. Eng. Sci., 64, 2433-2447 (2009).

5) S. A. K. Jeelani, R. Hosig, E. J. Windhab; Kinetics of low Reynolds number creaming and coalescence in droplet dispersions. AIChE J., 51, 149-161 (2005).
6) R. Matsuno, T. Yano; "Food physical chemistry (Shokuhin Butsurikagaku)”, Buneido, Tokyo, Japan, 1996, p. 135.

7) S. Adachi, J. Imagi, R. Matsuno; Model for estimation of the stability of emulsion in a creaming layer. Biosci. Biotech. Biochem., 56, 495-498 (1992).

8) E. J. W. Verwey, J. Th. G. Overbeek; "Theory of the Stability of Lyophobic Colloids”, Elsevier, Amsterdam 1948.

9) Y. Miyagawa, K. Katsuki, R. Matsuno, S. Adachi; Effect of oil-droplet size on activation energy for coalescence of oil droplets in an $\mathrm{O} / \mathrm{W}$ emulsion. Biosci. Biotechnol. Biochem., 79, 1695-1697 (2015).

10) von B. Derjaguin; Untersuchungen uber die Reibung und Adhasion, IV . Theorie des Anhaftens kleiner Teilchen. Kolloid Z., 69, 155-164 (1934).

11) H. C. Hamaker; The London-van der Waals attraction between spherical particles. Physica, 4, 1058-1072 (1937).

12) S. Rentsh, R. Pericet-Camara, G. Papastavrou, M. Borkovec; Probing the validity of the Derjaguin approximation for heterogeneous colloidal particles. Phys. Chem. Chem. Phys., 8, 2531-2538 (2006).

13) T. Mita. K. Yamada, S. Matsumoto, D. Yonezawa; Dispersion state of protein-stabilized emulsions. Dependence of globule size and size distribution upon $\mathrm{pH}$ in concentrated oil-inwater systems. J. Texture Stud., 4, 41-51, (1973). 